\title{
Synthesis and biological evaluation of curcumin-like diarylpentanoid analogues for anti- inflammatory, antioxidant and anti-tyrosinase activities
}

\begin{abstract}
A series of 46 curcumin related diarylpentanoidanalogues were synthesized and evaluated for their anti-inflammatory, antioxidant and anti-tyrosinaseactivities. Among these compounds 2, 13 and 33 exhibited potent NO inhibitory effect on IFN- $\gamma /$ LPS-activated RAW 264.7 cells as compared to l-NAME and curcumin. However, these series of diarylpentanoidanalogues were not significantly inhibiting NO scavenging, total radical scavenging and tyrosinase enzyme activities. The results revealed that the biologicalactivity of these diarylpentanoidanalogues is most likely due to their action mainly upon inflammatory mediator, inducible nitric oxide synthase (iNOS). The present results showed that compounds 2, 13 and 33 might serve as a useful starting point for the design of improved anti-inflammatory agents.
\end{abstract}

Keyword: Anti-inflammatory; Antioxidant; Anti-tyrosinase; Diarylpentanoids; iNOS. 